THE PERFORMANCE OF A SUPERCONDUCTING MICRO-UNDULATOR PROTOTYPE

I. Ben-Zvi, Z.Y. Jiang, G. Ingold, L.H. Yu, W. B. Sampson

May 1990

Work performed under the auspices of the U.S. Department of Energy, under contract no. DE-ACO2-76CH00016.

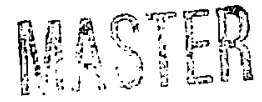




\title{
THE PERFORMANCE OF A SUPERCONDUCTING MICRO-UNDULATOR PROTOTYPE
}

\author{
I. Ben-Zvi, Z. Y. Jiang, G. Ingold and $\mathbb{L}$. H. Yu
}

NSLS Department

W. B. Sampson

ADD Department

Brookhaven National Laboratory

Upton, N.Y. 11973

\begin{abstract}
We report on the performance of a prototype of a super-ferric microundulator. The micro-undulator consists of a continuous winding of niobiumtitanium wire wound on a low carbon steel yoke. It is about 3 periods long with a period of $8.8 \mathrm{~mm}$ and a gap of $4.4 \mathrm{~mm}$. The undulator achieves the a peak magnetic field on axis of over 0.5 Tesla. Asymmetry of the field pattern due to a dipole component was identified, analyzed and a correction has been applied to the undulator ends to produce a symmetric field distribution. Within the precision of the measurement the field pattern produced by the super-ferric undulator needed no correction.
\end{abstract}




\section{MICRO-UNDULATORS}

As electron accelerators improve in their beam emittance one finds a need for shorter period undulators to take advantage of the low emittance and thus we may reduce the wavelength of Free Electron Lasers using these beams. The shortest wavelength an FEL may operate at, $\lambda_{\min }$, is proportional to the (un-normalized) beam emittance $\epsilon$,

$$
\lambda_{\min } \approx 2 \pi \epsilon
$$

We also have the FEL resonance condition

$$
\lambda=\frac{\lambda_{u}\left(1+a_{u}^{2}\right)}{2 \gamma^{2}}
$$

where $\lambda_{u}$ is the undulator wavelength and $a_{u}=0.66 B \lambda_{u}$ is the undulator parameter where $B$ is peak magnetic field of the undulator (on the beam axis) in Tesla and $\lambda_{u}$ is in $\mathrm{cm}$. Thus the undulator period is proportional to the emittance and square of the linac energy,

$$
\lambda_{u} \approx 4 \pi \gamma^{2} \epsilon /\left(1+a_{u}^{2}\right)
$$

We may express the emittance by the invariant $\epsilon_{n}=\gamma \epsilon$ and taus we get

$$
\lambda_{u} \approx 4 \pi \gamma \epsilon_{n} /\left(1+a_{u}^{2}\right) .
$$

Miicro-undulators will play an increasingly significant role in compact FELs based on low ernittance guns.

As an example, the Accelerator Test Facility at Brookhaven National Laboratory has a low-emittance laser-photocathode gun, $\epsilon_{n}=6 \times 10^{-6} \pi m$ Rad. This emittance makes it possible may build a $500 \mathrm{~nm}$ FEL with no more than $50 \mathrm{MeV}$ electron beam energy. The undulator period which is reeded is under $1 \mathrm{~cm}$, or what has been called a micro-undulator.

The undulator period which is suitable for this beam energy and emittance is 9 millimeters. Conventional techniques of undulator construction become increasingly difficult as the period is reduced below about $1 \mathrm{~cm}$. Furthermore, for fast tuning of the FEL wavelength an electromagnet has a great advantage. Pulsed electromagnets may degrade the FEL bandwidth unless the field is extremely well stabilized. Thus we find that a superconducting electromagnet has a number of advantages. 
After making the decision to use a superconducting magnet we are still left with the choice of an air core or a ferromagnetic core. An air core superconducting magnet can achieve a high magnetic field, but the magnetization of a ferromagnetic core can always enhance the field, even at saturation. The contribution of the core's magnetization becomes significant as we reduce the dimeusions of the undulator, since the current density in the wire becomes the dominant factor. This can be shown as follows:

The undulator parameter $a_{\mathrm{u}}$ is given as a function of the current and gap by the following approximate relationship [2]:

$$
a_{u}=610^{-4}(N I)\left[\frac{1}{\sinh (\xi)}-\frac{1}{\sinh (3 \xi)}\right]
$$

where $(N I)$ is the ampere turns per coil, $\xi=\pi g / \lambda_{u}$, and $g$ is the gap of the undulator. This expression holds below saturation only.

For a constant $\xi$ the undulator parameter depends only on (NI). However, as we scale down the dimensions of the undulator the space available for winding the coil decreases its area in proportion to $\lambda_{u}^{2}$, thus in microundulators current density is the dominant design limitation, and a below a certain period a super-ferric undulator will perform better than an air core undulator.

There are additional advantages to a super-ferric design. One is the precision which can be obtained. The ferromagnet field accuracy depends mostly on the geometry of the ferromagnet, which can be precision machined inexpensively, and not so much on the wire placement, which is inherently a less accurate construction process. For a given undulator field the wire is immersed in a lower magnetic field in a ferromagnet, thus we operate with a less critical superconducting design. 


\section{THE SUPER-FERRIC MICRO-UNDULATOR DESIGN}

The design of the undulator is shown in Fig. 1. It consists of two low carbon-steel yokes $61.6 \mathrm{~cm}$ long, $8.3 \mathrm{~mm}$ high and $15.9 \mathrm{~mm}$ wide. The undulator period is $8.8 \mathrm{~mm}$, and the full gap (the separation between the yokes where the beam passes) is $4.4 \mathrm{~mm}$. The wire is wound in the $2.2 \mathrm{~mm}$ wide channels. The design will use 18 turns in each gap, in four layers. Fig. 1 shows the notches in between channels which allow the passage of the wire from one channel to the next. Thus we use a continuous winding along the whole undulator. This contributes to the precision of the field values along the undulator and makes it possible to run the undulator in a persistent current mode.

The number of turns per layer alternates between five and four turns, so that the notch between a pair of wires in a layer serves as a guide for the wire above or below that layer.

The wire we use is a 54 filament niobium-titanium superconductor with a copper to superconductor ratio of 1.3 [3]. The wire diameter (with formvar insulation) is $0.044 \mathrm{~mm}$. The critical current as specified by the manufacturer is 142 amperes at 5 Tesla (4.2K). The short sample critical current of this wire has been measured as a function of the applied (external) magnetic field. The result is shown in Fig. 2 (open circles). As can be seen from this measurement, the manufacturer's specification for the critical current is met.

We are using an even number of layers and a winding scheme in which every layer is wound along the whole yoke, reversing direction at each pole. This scheme has the advantage that both ends of the coil exit at the same end of the yoke, making the elimination of stray fields from the connections easy.

In the assembled undulator the two yokes are to be held at a precise separation by a stainless steel holder. The electron beam will pass in a thin wall stainless steel tube heid by the same holder at the center of the undulator. This beam tube will provide the vacuum tight boundary of the liquid helium, thus the yokes and wire will be immersed in the liquid helium cryogen.

Simulation of the undulator was carried out using the program POISSON [4]. This is a two-dimensional calculation but for this particular undulator geometry one expects a good agreement between the simulation and reality.

In Fig. 3 we show the magnetic field lines as calculated by the program 
at a current of 2800 ampere-turns. As this figure shows, the magnetic field on the wire is not uniform. The peak magnetic field on the wire is at the corner of the layer, next to the sharp end of the ferromagnetic 'tooth'.

The value of this peak wire magnetic field is plotted vs. the current in the wire by the solid circles of Fig. 2. We expect the magnet to quench as the peak magnetic field vs. current curve intersects the critical current vs. the (external) magnetic field curve, i.e., at a current of about 230 amperes for this particular magnet (with 14 wires per groove).

The simulation predicts a peak axial magnetic field of $0.475 \mathrm{~T}$ for a current of NI=2800 ampere-turns. This corresponds to an $a_{u}$ of 0.28 . To achieve this current using 14 turns we need 200 amperes, safely below the 230 ampere limit. 


\section{SUPERCONDUCTING TEST RESUITS}

A short sample of the undulator was built and tested. The sample has a few deviations from the final undulator design since the experience gained in the construction of the test piece served to improve the design. Thus the test piece has only three layers. It was originally $19.8 \mathrm{~mm}$ long, with four $2.2 \mathrm{~mm}$ gaps for winding the wire and five $2.2 \mathrm{~mm}$ wide poles. The height of the test piece yoke is $6.4 \mathrm{~mm}$, its width $12 \mathrm{~mm}$ and the undulator period 8.8 $\mathrm{mm}$. Each gap was wound with 14 turns $(=5+4+5)$ of the above mentioned wire. The winding was not poited with epoxy.

The two yokes were assembled on a brass holder and mounted in a test cryostat. A cryogenic Hall probe was guided at the center of the test piece undulator by a G10 form. The position of the probe along the test piece was determined by a precision translator. The probe reading was digitized and shown vs. probe position on a computer graphic display.

The result of the first measurement is shown in Figure 4. One may notice that the field pattern of the undulator has a dipole component, that is to say it looks like a sine function plus a constant.

We have interpreted the asymmetry as an edge effect. Simulations done with POISSON have shown that the asymmetries may be reduced, even inverted, by changing the reluctance at the edges of each yoke and also possibly change the current of the two extreme windings of each yoke. To test this numerical simulation result we have modified the micro-undulator test piece, adding a 'quarter period' at each end. The form on which these additional coils were wound was made from a non magnetic material, but the pole was made from the same 1006 low carbon steel. We have provided for more turns in these correction-coils to allow for a range of ampere turn values both above and bellow the main coil.

The results of the test of this micro-undulator test piece are shown in Figs. 5,6 and 7. In Fig. 5 we see the magnetic field on axis as a function of position along the undulator axis, when the central coils are run at 100 amperes and the end coils (the correction quarter periods) are run at 140.7 amperes. This choice of correction coils' current was found empirically by looking for a zero dipole moment. Thus we show that the dipole component indeed can be corrected at the end of the undulator.

Fig. 6 presents the same information as the previous figure but with 180 amperes at the central coils and 200 amperes at the end coils. The peak magnetic field on axis is 0.437 Tesla. 
The quench currents of this coil are as follows: with center coils only, 261 amperes; with end coils only, 228 amperes; with all coils run in series, 232 amperes. This result is in good agreement with the prediction based on the short sample critical current measurement and the POISSON calculation, as presented in Fig. 2.

In Fig. 7 we show the currents at the center coils and the end coils for a balanced (no dipole component) operation of the undulator test piece as a function of the peak magnetic field on axis. For the purpose of comparison to excitation curves, the magnetic field is shown on t!: vertical scale, however one should read Fig. 7 in the following way: First one selects a field value on the vertical scale. Proceed horizontally to intersect the curves. The intersection points indicate the currents in the center coils and in the end coils which produce the magnetic field value in a balanced mode.

One can see that about $5 \mathrm{kG}$ the balanced undulator needs nearly equal currents in the central and end-coils of about 215 amperes, within the quench current limit of both center and end coils.

In Fig. 7 we also show the POISSON calculation for the peak field vs. current (open triangles). This is a simulation of an infinitely long undulator, thus the center coil and end coils are merged. The theoretical curve matches well the average of the two experimental curves. Although not shown, saturation is expected to begin â about 200 amperes. 


\section{REFERENCES}

1. K. Batchelor et. al., A Visible FEL at the Brookhaven Accelerator Test Facility, to be published in Nucl. Instr. and Meth. A.

2. S. Krinski, Brookhaven National Lab.BNL 25698, (1979)

R.P. Walker, Nucl. Instr. and Meth. A237,366(1985).

3. Supplied by Oxford Superconducting Technology, Carteret, NJ.

4. Poisson Group of Codes, Los Alamos, LA-UR-78-115 (1987).

\section{FIGURE CAPTIONS}

1. Super-ferric micro-undulator design.

2. Open circles: the measured short sample critical current vs. applied magnetic fielc of the wire used in the super-ferric micro-undulator. Solid circles: POISSON calculation of the peak magnetic field on the wire in the micro-undulator vs. the wire in the current.

3. Magnetic field lines of the micro-undulator from the simulation by the two-dimeusional program POISSON.

4. The test undulator field (kGauss) vs. position (mm) at the initial configuration (no correction coils) and at 200 ampere turns.

5. Field on axis vs. position for the test piece with the correction coils. The current is 100 ampere in the center coils and 140.7 ampere in the end coils.

6. Field on axis vs. position for the test piece with the correction coils. The current is 180 ampere in the center coils and 200 ampere in the end coils.

7. Excitation current for the center coils (open circles) and for the end coils (full circles) are shown on the horizontal scale vs. the peak magnetic field on axis, shown on the vertical scale. The ratio of the center to end coil currents is selected to eliminate the dipole component.

The peak field on axis vs. current for an infinitely long micro-undulator from the POISSON simulation is shown by open triangles. 


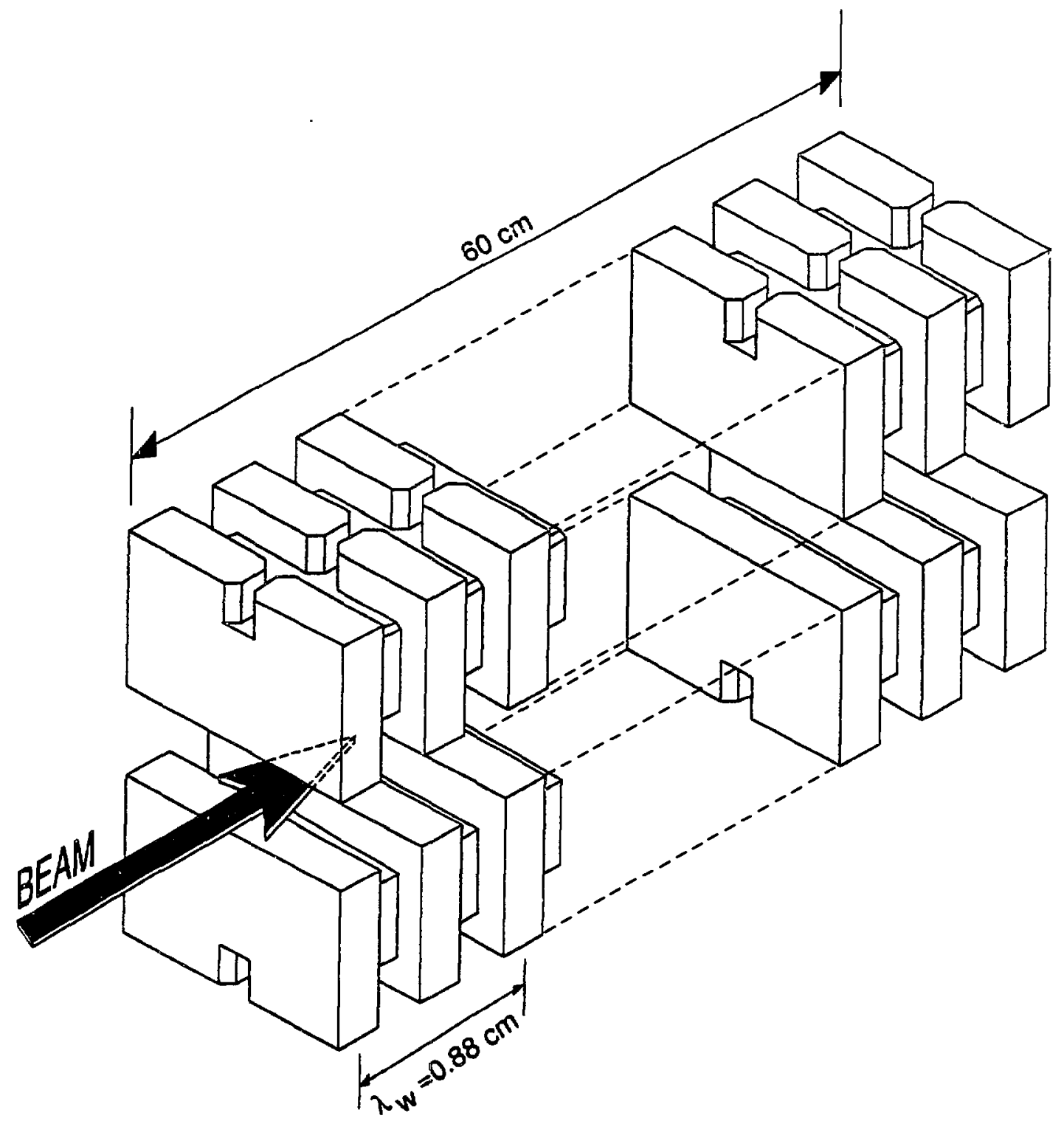




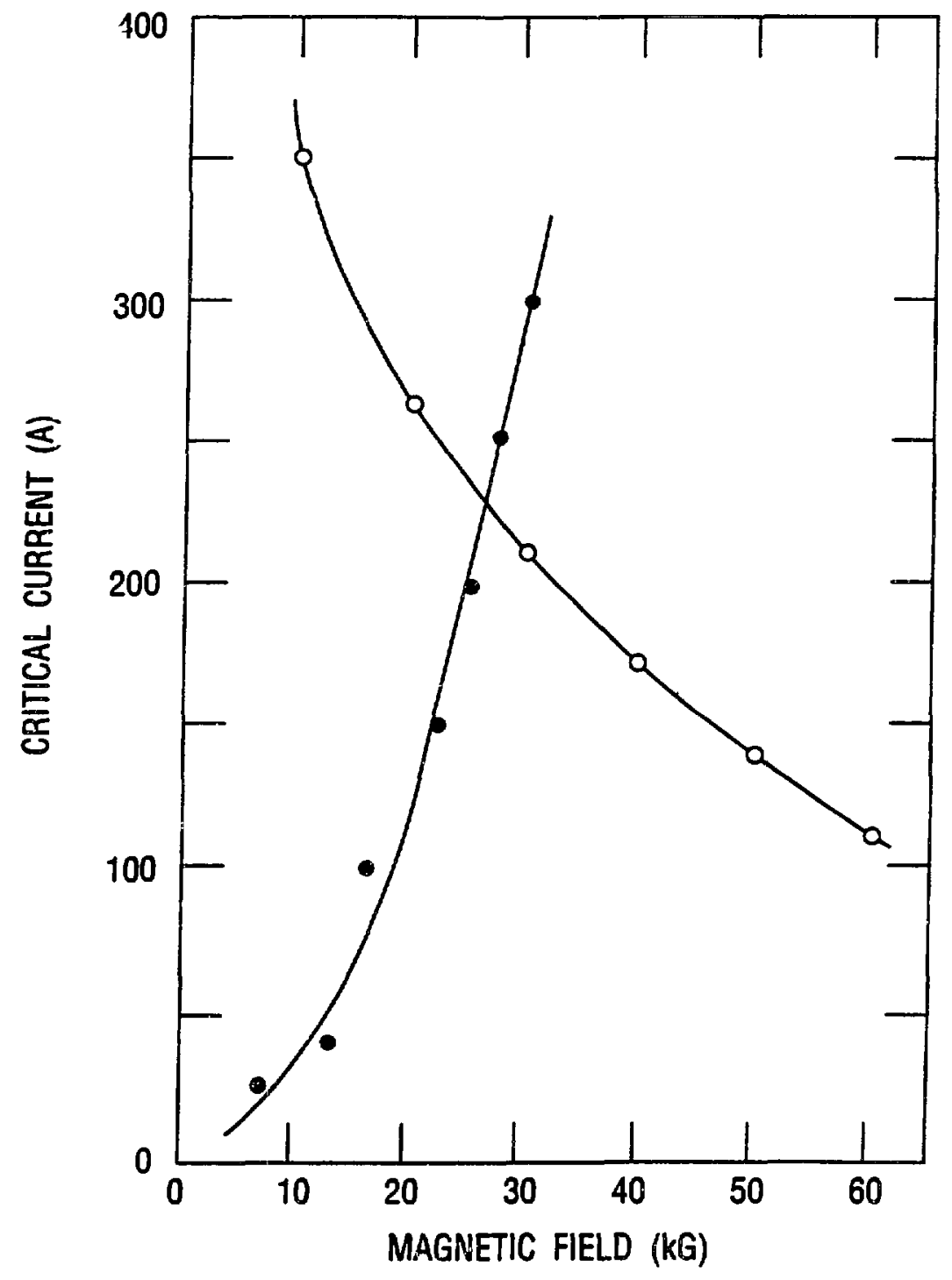




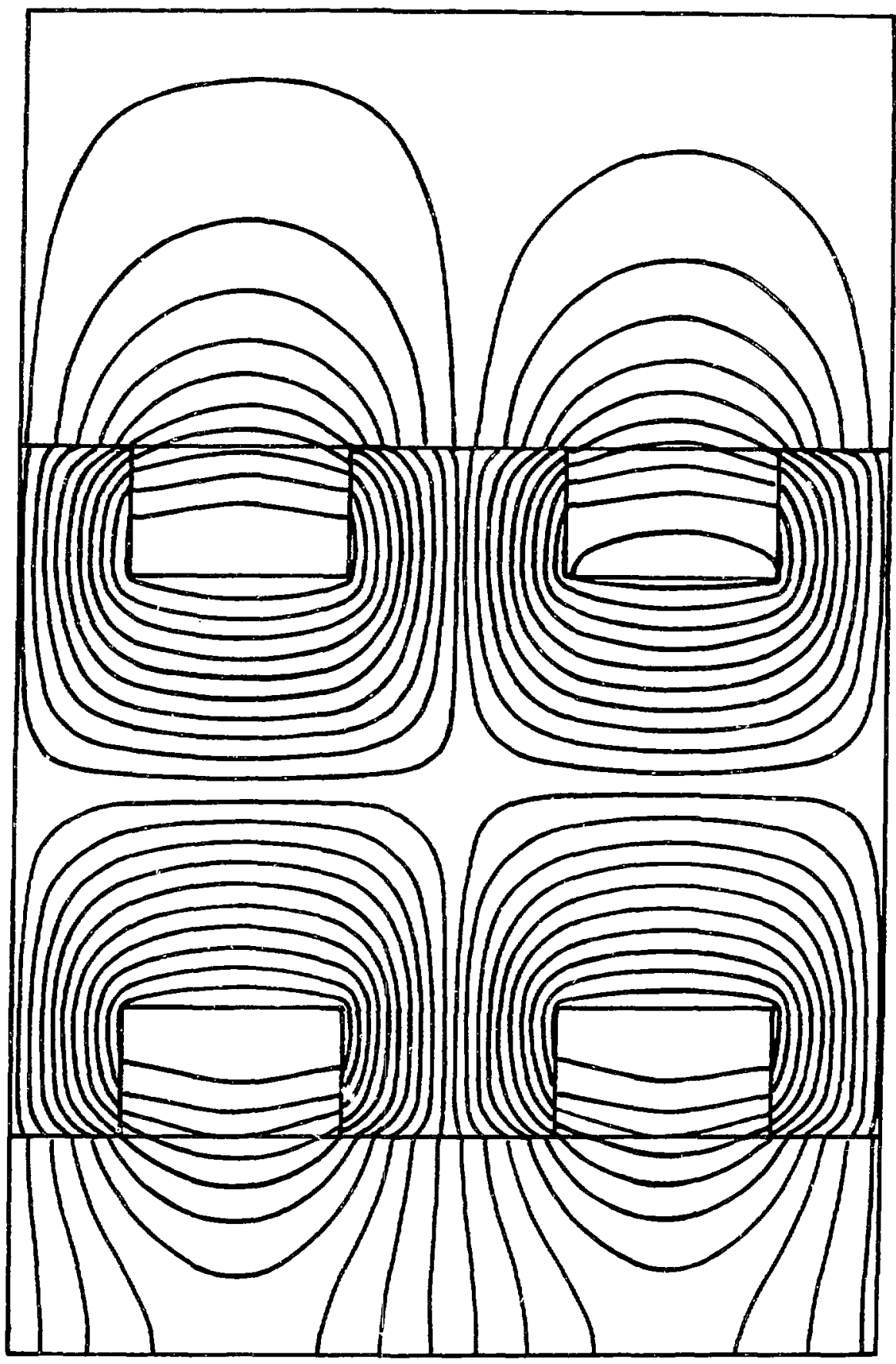




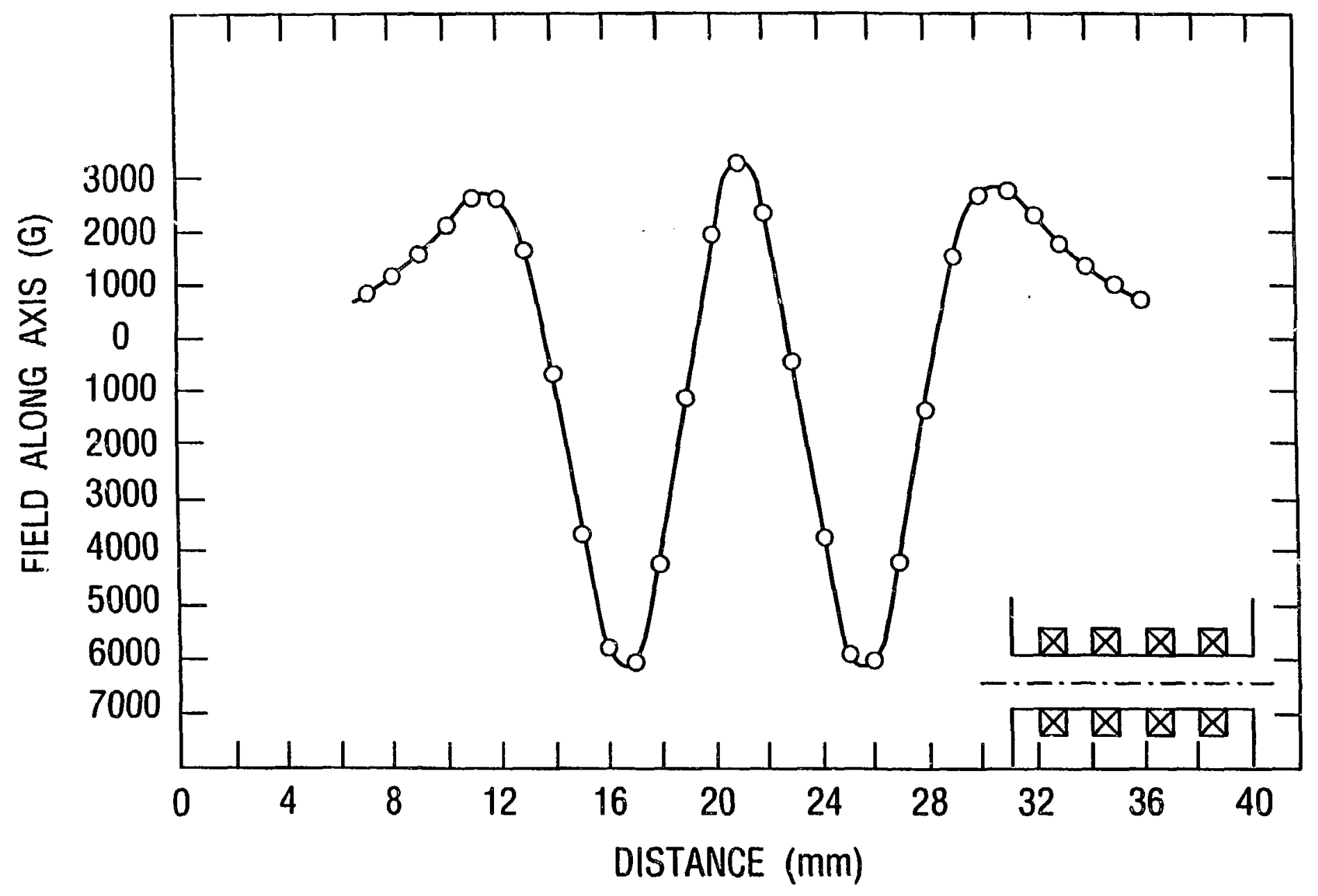




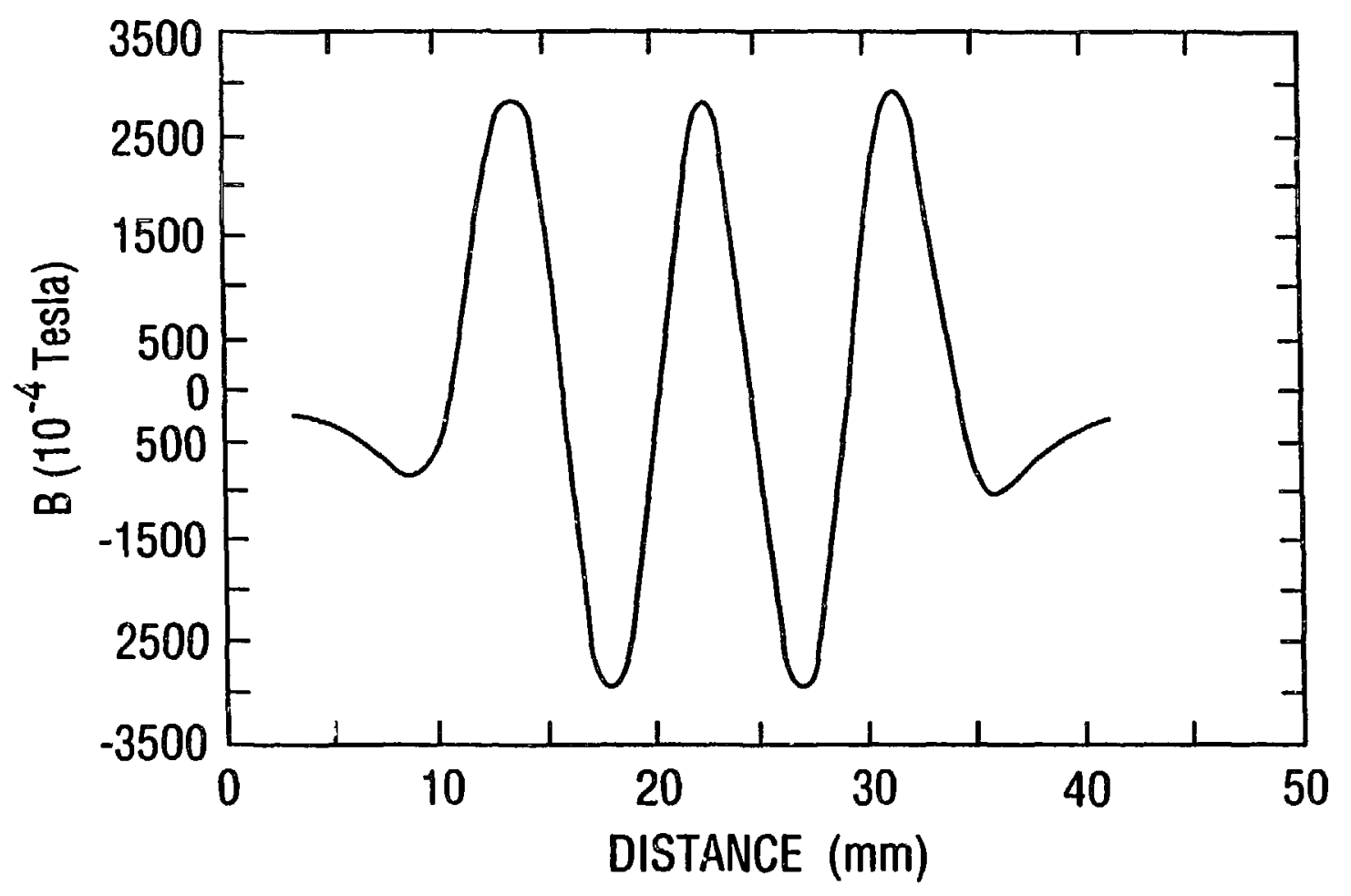




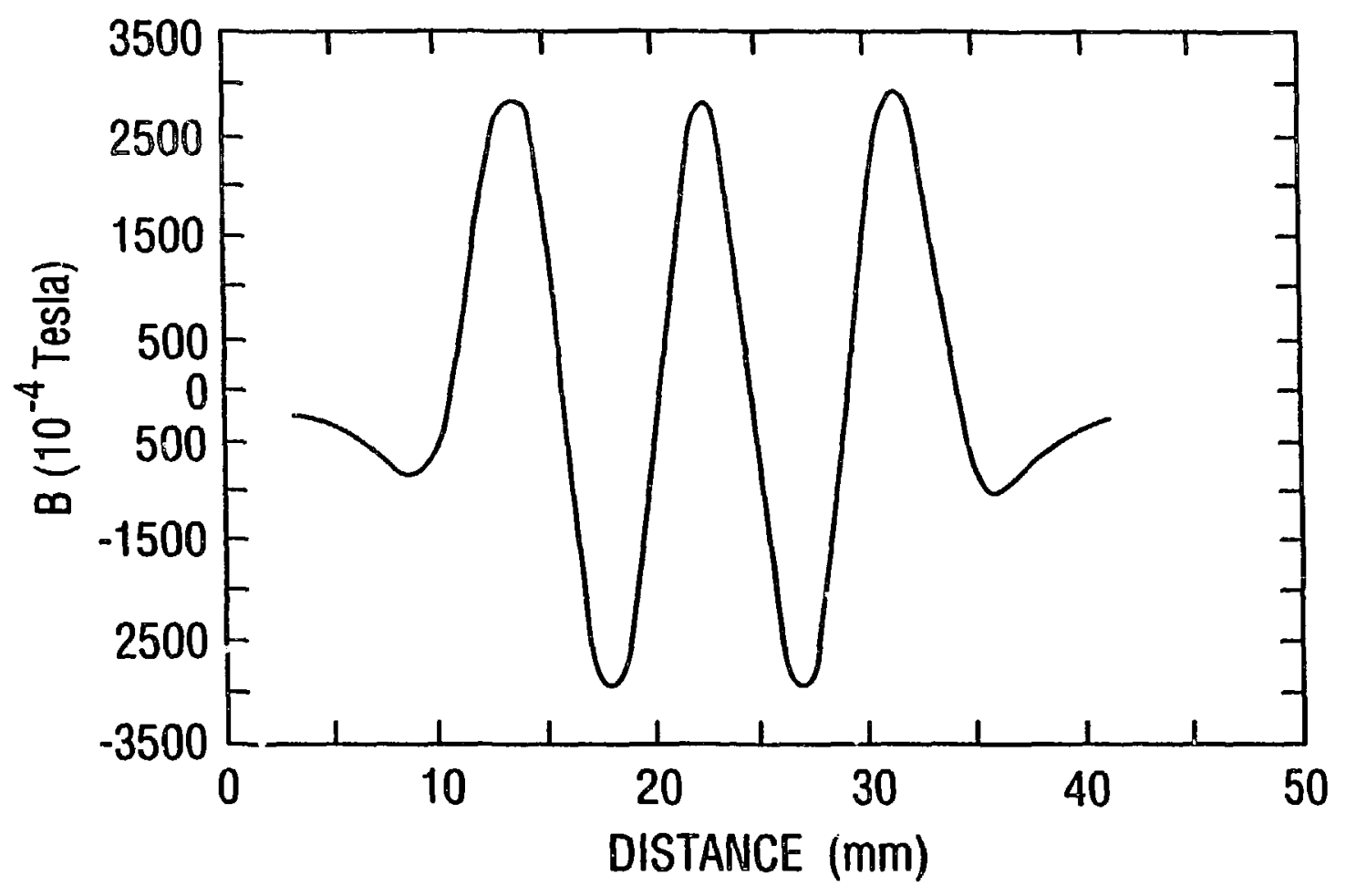




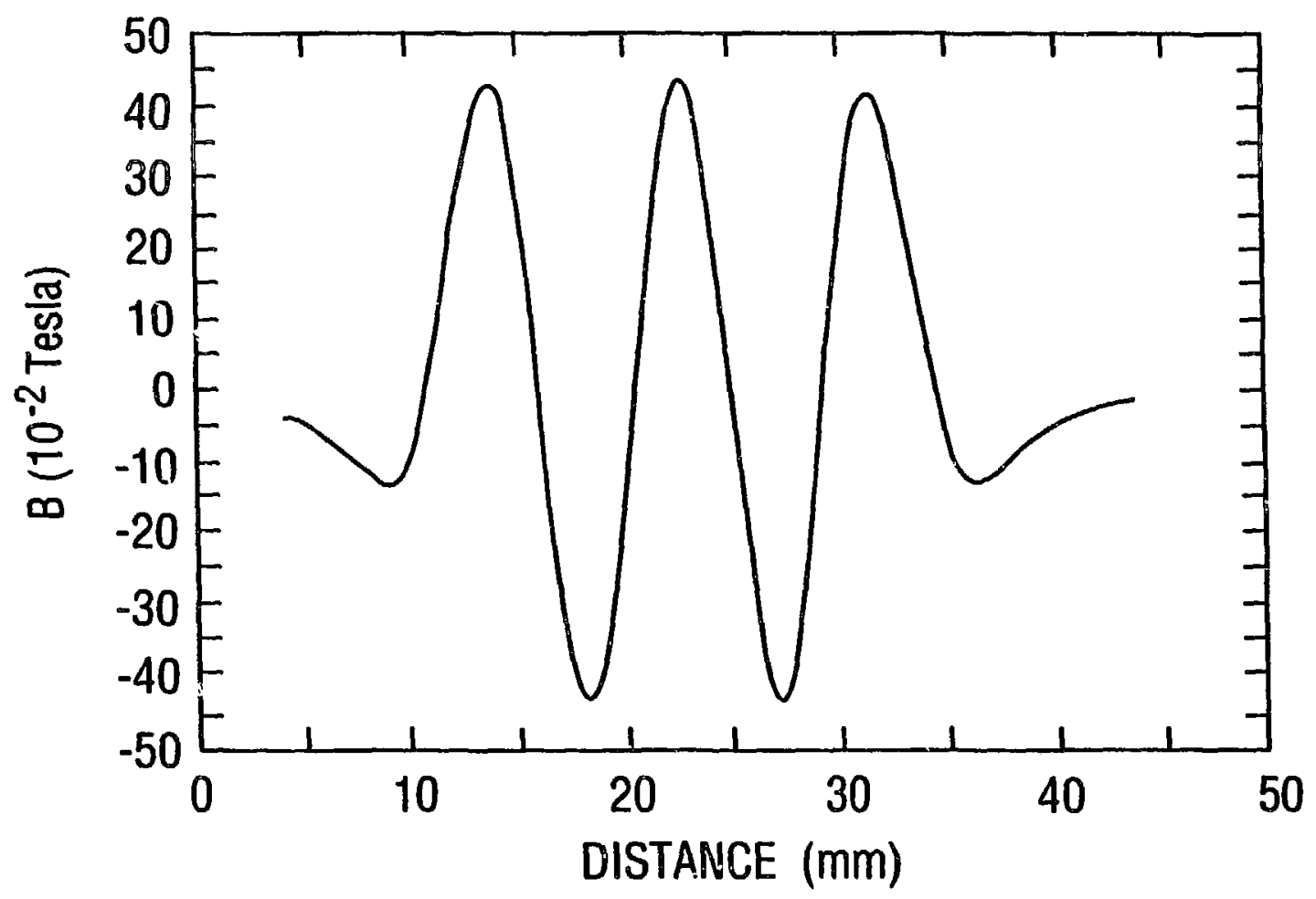




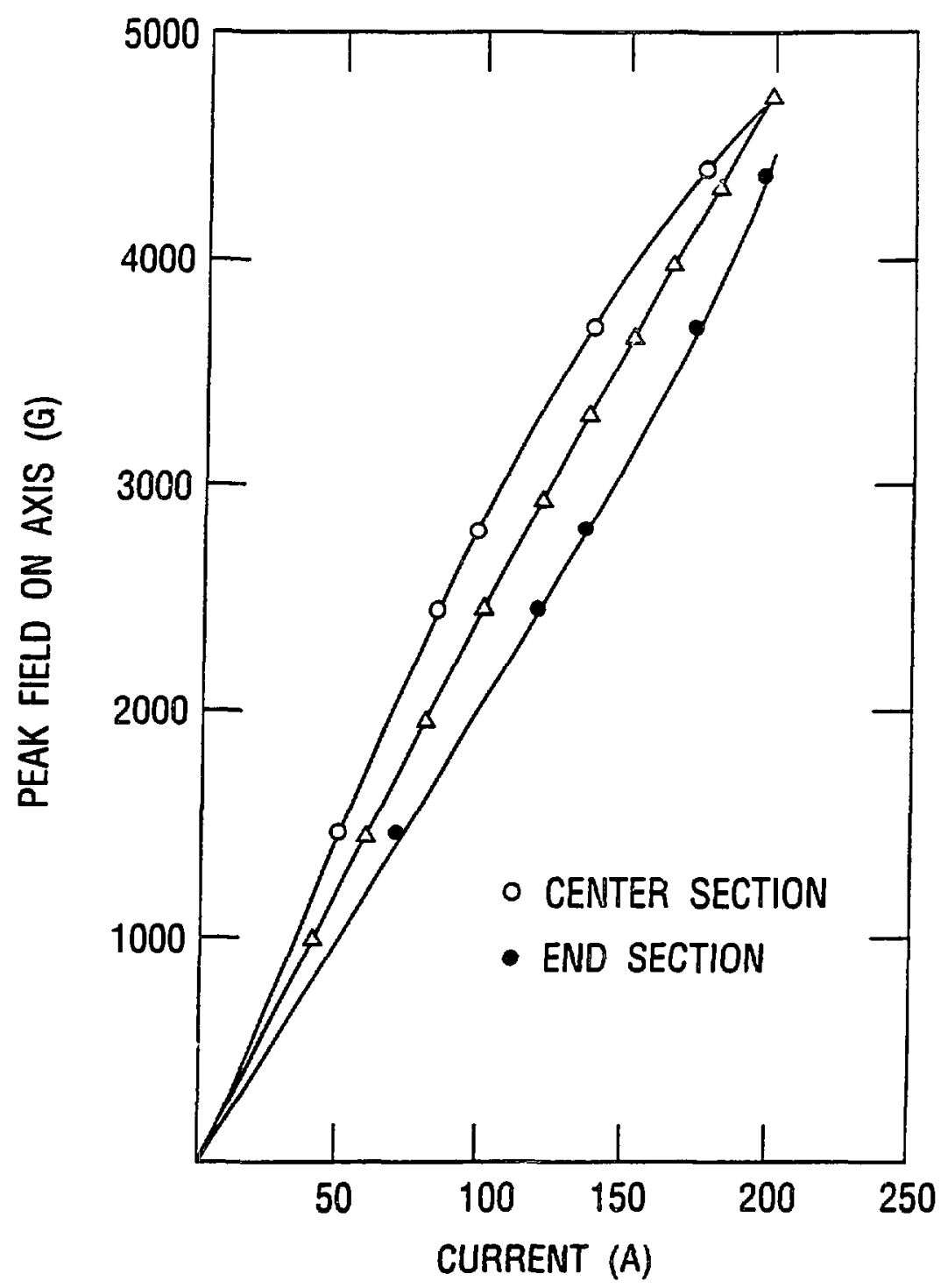

